\title{
Nasal chemosensory tests: biomarker between dementia with Lewy bodies and Parkinson disease dementia*
}

\author{
Clovis Foguem ${ }^{1,2}$, Mohamed Lemdani ${ }^{3}$, Caroline Huart ${ }^{4,5}$ \\ 'Auban Moët Hospital, 137 rue de l'hôpital, 51200 Epernay, France \\ 2 University of Burgundy, 21000 Dijon, France \\ ${ }^{3}$ Faculty of Pharmaceutical and Biological Sciences, EA 2694, University of Lille, 3, rue du Professeur Laguesse, 59006 Lille, France \\ ${ }^{4}$ Department of Otorhinolaryngology, Cliniques universitaires Saint-Luc, Brussels, Belgium \\ ${ }^{5}$ Institute of Neuroscience, Université catholique de Louvain, Brussels, Belgium
}

Rhinology 58: 6, 605 - 609, 2020

https://doi.org/10.4193/Rhin20.072

* Received for publication:

February 29, 2020

Accepted: May 26, 2020

\begin{abstract}
Background: Dementia with Lewy bodies (DLB) and Parkinson disease dementia (PDD) are progressive and disabling neurodegenerative disorders, which are often misdiagnosed due to theirs overlapping clinical and paraclinical features. Nevertheless, their adequate management requires an accurate differential diagnosis. The main aim of this study was to investigate the usefulness of olfactory and trigeminal nasal testing for the differential diagnosis between DLB and PDD.
\end{abstract}

Methods: Odor thresholds to three odorants differentially activating the olfactory and trigeminal systems were assessed in patients with DLB, PDD and healthy controls ( $n=20$ per group).

Results: Odor thresholds were significantly different between the three groups of subjects. More precisely, we found that DLB patients had significantly lower detection threshold performances compared to PDD patients. Moreover, using a standard canonical discriminant analysis, we confirmed a plain differentiation between the three groups.

Conclusions: The current study highlights that DLB patients have very poor olfactory and trigeminal detection threshold performances, which are significantly lower, compared to PDD patients. These results suggest that olfactory testing, using odorants that stimulate both the olfactory and trigeminal systems, could constitute an interesting biomarker and contribute to the differential diagnosis of PDD and DLB patients. Further researches, notably on olfacto-trigeminal interactions, are warranted in these populations to support our findings.

Key words: biomarker, dementia with Lewy bodies, differential diagnosis, odor detection thresholds, Parkinson disease dementia

\section{Introduction}

Dementia with Lewy bodies (DLB), Parkinson disease (PD) and Parkinson disease dementia (PDD) are part of synucleinopathies ${ }^{(1,2)}$. Clinically, DLB and PDD have overlapping clinical appearance, with similar symptoms (including mainly parkinsonism, fluctuating cognition, and visual hallucinations) ${ }^{(3,4)}$. Consequently, their differential diagnosis is often challenging and DLB is often misdiagnosed ${ }^{(1,5)}$, despite the revised 2017 DLB's $^{\prime}$ diagnostic criteria ${ }^{(2)}$.

Currently, the temporal course of the emergence of the symptoms is used to differentiate between these two diseases. DLB is suspected when dementia precedes or coincides with the occurrence of parkinsonism or appears within the year following the development of parkinsonism. On contrast, PDD is suspected when dementia appears in the context of a previously known PD. However, this "temporal rule" is controversial, since some DLB patients may present with motor symptoms first. Early diagnosis is crucial to ensure adequate follow-up, counseling and treatment. Since patients' management is not the same for these diseases, it is therefore crucial to develop reliable and accurate biomarkers for their differential diagnosis. Some ongoing researches aim to establish blood, cerebrospinal fluid or imaging biomarkers of PDD and DLB ${ }^{(6)}$; however, their availability is limited and results are not yet formally conclusive. 
Table 1. Main demographic and clinical data of patients with Dementia with Lewy bodies (DLB) and matched Parkinson disease dementia (PDD) patients or healthy controls and odor (PEA, n-butanol and pyridine) metric characteristics.

\begin{tabular}{|c|c|c|c|}
\hline $\begin{array}{l}\text { Groups [20 subjects / Group] } \\
\text { (mean age } \pm \text { SD [min, max]) }\end{array}$ & $\begin{array}{c}\text { Dementia with Lewy } \\
\text { bodies (DLB) } \\
(86.00 \pm 6.32 \\
{[69-97] \text { years old) }}\end{array}$ & $\begin{array}{c}\text { Disease Dementia (PDD) } \\
(77.85 \pm 7.11 \\
{[65-90] \text { years old) }}\end{array}$ & $\begin{array}{c}\text { Healthy controls } \\
(85.65 \pm 6.19 \\
{[65 \text { - 93] years old) }}\end{array}$ \\
\hline PEA (mean dilution \pm SD $[\min , \max ]$ ) & $1.95 \pm 3.75[0,13]$ & $14.20 \pm 6.53[2,23]$ & $21.20 \pm 3.59[0,23]$ \\
\hline $\mathrm{n}$-Butanol (mean dilution $\pm \mathrm{SD}[\min , \max ])$ & $2.65 \pm 4.09[0,15]$ & $11.40 \pm 6.37[0,20]$ & $19.05 \pm 1.90[0,20]$ \\
\hline Pyridine (mean dilution \pm SD [min, $\max ]$ ) & $3.65 \pm 3.01[0,11]$ & $20.20 \pm 5.57[6,26]$ & $24.45 \pm 4.13[0,26]$ \\
\hline $\begin{array}{l}\text { Duration of the illness (DLB or PDD) } \\
\text { (mean duration } \pm \mathrm{SD}[\mathrm{min}, \mathrm{max}] \text { ) }\end{array}$ & $02.09 \pm 2.38[0,11]$ & $11.35 \pm 8.34[5,34]$ & \\
\hline $\begin{array}{l}\text { Mini-mental state examination score } \\
\text { (mean score } \pm \text { SD }[\min , \max ] \text { ) }\end{array}$ & $17.50 \pm 2.70[15,23]$ & $20.00 \pm 2.94[16,27]$ & $23.15 \pm 3.38[15,28]$ \\
\hline
\end{tabular}

Main demographic and clinical data are presented as average \pm standard deviation $(\mathrm{SD})$ and extreme values $(\mathrm{minimum}=$ min; maximum $=$ max).

It is widely acknowledged that olfactory dysfunction is present in various neurological diseases (7), among which PD, PDD, Alzheimer's disease (AD) ${ }^{(8)}$ and DLB. However, the trigeminal function, although being involved in odors perception ${ }^{(9)}$ is sparsely explored in these diseases. In the present study, we aimed, to investigate whether global odors perception (olfactory or trigeminal functions) test could be a useful for the differential diagnosis between DLB and PDD. For this purpose, we assessed nasal detection thresholds for three odorants which differentially activate the olfactory and/or trigeminal systems. In addition, we aimed to determine whether the trigeminal function is also affected in DLB or PDD.

\section{Materials and methods}

Ethical standards

Sixty participants distributed into three groups (20 DLB ${ }^{(10)}, 20$ PDD (11) and 20 matched healthy Controls) underwent odors detection thresholds testing using Phenylethyl alcohol (PEA), n-Butanol and Pyridine thresholds. The study was conducted according to the Helsinki's declaration and was approved by the local Institutional Ethics Review Board. Written informed consent was obtained from all the participants.

\section{Participants}

60 elderly participants $(83.17 \pm 7.48$ [65; 97] years old) were included in the present study: 20 DLB patients ( 12 female), 20 gender-matched moderate PDD patients and 20 healthy controls (Table 1). Overall, these 3 groups differed significantly according to age $(p<0.05)$, although DLB patients and healthy controls group did not differ $(p=0.984)$.

The participants had no history of sinonasal diseases, head injury or stroke prior to the olfactory tests and were all nonsmokers. Additionally, participants were free from patent depression symptoms [assessed by Mini-Geriatric Depression Scale (Mini-GDS)] ${ }^{(12)}$. Moreover, control participants were free from neurological disease and had no signs of Parkinsonism. The main demographic and clinical variables of the 3 groups of participants are described in Table 1.

Neurocognitive tests were performed to detect cognitive impairment using Mini-mental state examination (MMSE) ${ }^{(13-15)}$ and the clock-drawing test ${ }^{(16)}$. Exclusion criteria were 1 ) incorrect clockdrawing test ( 0 or 1 out to 2); and/or 2) MMSE < 15/30 in patients and MMSE $<24 / 30$ in controls.

\section{Odors detection testing}

Odors detection thresholds were determined for three odorants known to differentially activate the olfactory and trigeminal systems based on the previous data ${ }^{(17)}$. Phenyl-ethyl alcohol (PEA) was chosen to activate almost exclusively the olfactory system; n-Butanol was considered to activate both the olfactory and trigeminal systems ${ }^{(18)}$. Finally, Pyridine was used to be specifically activating trigeminal afferents ${ }^{(19)}$. Patients were assessed following methods described in our previous publications ${ }^{(18,20)}$. In summary, odor thresholds were assessed using an ascending staircase, binary (stimulus vs. blank) forced-choice procedure, with inter-trial intervals of 90 seconds. The dilution step at which the odorant stimulus was first detected correctly three times in a row was recorded as the detection threshold ${ }^{(20)}$. The possible threshold detection scores ranged from 0 to 20 for $n$-Butanol, 0 to 23 for PEA and 0 to 26 for Pyridine; with higher scores indicating better olfactory function.

\section{Data analysis}

Statistical analyses were performed using Statview (SAS Institute Inc., Statistical Software vendor_Version 9.2) and SPSS version 19.0 (IBM, Chicago, IL, USA). As variables were not normally distributed, according to the Shapiro-Wilk test, nonparametric Mann-Whitney-Wilcoxon test (two-tailed) or if needed, KruskalWallis test (with post-hoc tests) were used to compare odor thresholds. Spearman correlation analyses between odor thres- 
Table 2. [PEA, n-Butanol and Pyridine] odor detection thresholds comparison between Dementia with Lewy bodies (DLB) patients and matched healthy controls; between Parkinson disease dementia (PDD) patients and matched healthy controls; and between DLB patients and matched PDD patients.

\begin{tabular}{|c|c|c|c|c|}
\hline \multicolumn{2}{|c|}{$\begin{array}{l}\text { Comparison of odor detection thresholds (odor } \\
\text { thresholds) [PEA, } n \text {-Butanol and Pyridine] according } \\
\text { to Mann-Whitney-Wilcoxon test }\end{array}$} & \multirow{2}{*}{$\begin{array}{l}\text { Odor detection thresholds' } \\
\text { median [min; max] } \\
\qquad 11.00[0 ; 23]\end{array}$} & \multirow{2}{*}{$\begin{array}{l}\text { Estimated odor thresholds } \\
\text { quartiles values } \\
\text { (Q1 to } 25 \% / \text { to } 75 \% \text { Q3) } \\
0.00 / 22.75\end{array}$} & \multirow{2}{*}{$\begin{array}{l}\text { p-value } \\
0.001\end{array}$} \\
\hline between Dementia with & PEA thresholds & & & \\
\hline $\begin{array}{l}\text { Lewy bodies }(n=20) \text { and } \\
\text { healthy controls }(n=20)\end{array}$ & n-Butanol thresholds & $13.50[0 ; 20]$ & $0.00 / 20.00$ & 0.001 \\
\hline & Pyridine thresholds & $9.50[0 ; 26]$ & $3.00 / 26.00$ & 0.002 \\
\hline \multirow{3}{*}{$\begin{array}{l}\text { between Parkinson } \\
\text { disease dementia }(n=20) \\
\text { and healthy controls } \\
(n=20)\end{array}$} & PEA thresholds & $20.00[2 ; 23]$ & $11.5 / 23$ & $<0.001$ \\
\hline & n-Butanol thresholds & $18.00[0 ; 20]$ & $12.25 / 20$ & 0.011 \\
\hline & Pyridine thresholds & $25.00[6 ; 26]$ & $20.25 / 26.00$ & $<0.001$ \\
\hline \multirow{3}{*}{$\begin{array}{l}\text { between Parkinson } \\
\text { disease dementia }(n=20) \\
\text { and Dementia with Lewy } \\
\text { bodies }(n=20)\end{array}$} & PEA thresholds & $6.00[0 ; 23]$ & $0.00 / 14.5$ & 0.008 \\
\hline & n-Butanol thresholds & $6.00[0 ; 20]$ & $0.00 / 13.00$ & 0.054 \\
\hline & Pyridine thresholds & $9.00[0 ; 26]$ & $3.00 / 21.00$ & 0.001 \\
\hline
\end{tabular}

$\mathrm{n}=$ number of participants.

holds and clinical data were performed. Multivariate analyses were conducted to take into account the effect of some co-variables (age, gender, MMSE and stimuli's order of presentation) on the odor detection performances of the different groups. Finally, a discriminant analysis (with main components) was performed to evaluate the possibility to predict the participants' status (DLB, PDD and controls) based on the odor detection thresholds. The alpha level was set at 0.05 .

\section{Results}

Comparison of odor detection thresholds

Odor detection performances to the three odorants (PEA, nButanol and Pyridine) significantly differed between the three groups $(\mathrm{P}<0.001)$. Post hoc pairwise comparisons showed that all detection performances were significantly better in healthy controls compared to DLB $(P \leq 0.002)$ and to PDD $(P \leq 0.011)$. PEA and Pyridine detection thresholds performances were significantly lower in the DLB than in the PDD groups ( $\mathrm{P}=0.008$ and $P=0.001$, respectively) (Table 2 ), even after controlling for age. While $n$-Butanol detection thresholds performances were close to significance level $(P=0.054)$ (Figure 1).

Odor performances were not affected by gender $(P=0.547$, $\mathrm{P}=0.765$ and $\mathrm{P}=0.681$, for $\mathrm{PEA}, \mathrm{n}$-Butanol and Pyridine, respectively). Multivariate analysis found that the significant differences in odors detection thresholds between groups remained even after controlling for age and sex.

\section{Correlation analysis results}

All odors detection thresholds performances were significantly correlated [PEA- $n-B u t a n o l(P<0.001, r=0.83)$; PEA-Pyridine $(P<0.001, r=0.80)$; and $n-B u t a n o l-P y r i d i n e ~(P<0.001, r=0.76)]$.

\section{Discriminant analysis}

A factorial discriminant analysis was performed to classify participants based on a linear combination of the odors thresholds (PEA, n-Butanol and Pyridine). The results allowed for building two discriminating and distinct linear functions (functions scores). For a given participant, theses score functions are calculated using the following equations:

- Score $1=0.760 *$ PEA $+0.430 * n$-Butanol $+0.159 *$ Pyridine -3.966 ;

- Score $2=0.029 * P E A+0.203^{*} n-B u t a n o l-0.175^{*}$ Pyridine +0.208 . The group centroids, linear combination of the means for each odor threshold (PEA, n-Butanol and Pyridine), revealed a clear split between the three groups (DLB patients, PDD patients, healthy controls participants). Centroid plots, the resultant of the group centroids plotting are represented in Figure 2.

\section{Discussion}

In the present study, we found that DLB and PDD patients have significantly lower odor detection threshold performances compared to healthy controls; and that DLB patients perform significantly worse than PDD patients, independently of MMSE and age. We found that olfactory performances were not influenced by sex. This is congruent with the results of a recent study and a meta-analysis that found no gender-related differences when performing olfactory tests in people aged $>50$ years ${ }^{(21,22)}$. Discriminant analyses were able to discriminate between the three groups of participants based on odor detection thresholds to PEA, n-Butanol and Pyridine. This suggests that odor detection thresholds tests using these odorants could be useful biomarkers to discriminate DLB and PDD patients. Moreover, we noted that DLB patients performed significantly worse than PDD patients. This might suggest greater olfactory or trigeminal pathways impairment in DLB patients. This also would have an im- 
PEA

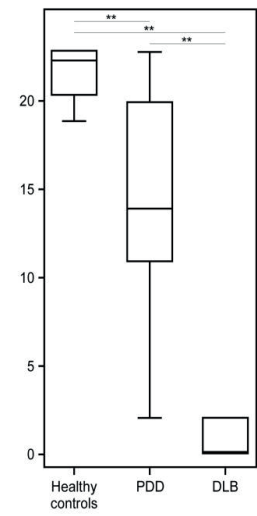

BUT

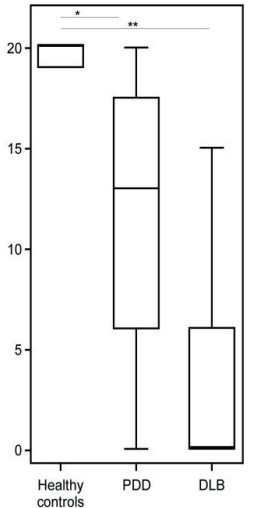

PYR

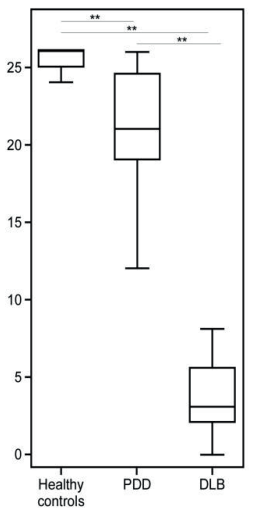

Figure 1. Average means odor [PEA, n-butanol and pyridine] detection thresholds comparison between Dementia with Lewy bodies' patients (DLB) and Parkinson disease dementia patients (PDD) and matched Healthy controls. $P$ value significance set at $<0.05$. Symbol meaning: * $\mathrm{P} \leq 0.05 ;{ }^{* *} \mathrm{P} \leq 0.01 ;{ }^{* * *} \mathrm{P} \leq 0.001$. Acronyms odorants: PEA: Phenyl ethyl alcohol; BUT: n-Butanol; PYR: Pyridine.

pact on the etio-pathogenesis of these two synucleinopathies. Notably, since odor thresholds are thought to reflect peripheral olfactory processing, our results suggest that abnormal protein deposition (mainly alpha-synucleinopathy in these cases) occur at least partly at the level of the olfactory neuroepithelium and olfactory bulb; and that these structures are more severely involved in DLB.

Several biomarkers are currently used for the diagnosis of synucleinopathies (i.e. MRI, PET, ${ }^{123}$ I-metaiodobenzylguanidine cardiac scintigraphy, REM sleep behavior disorder, serum or CSF biomarkers, genetics...). However, correct differential diagnosis remains challenging. Our results suggest that assessment of odor detection thresholds should be considered as a potential biomarker of DLB. Notably, its ability to discriminate between DLB and PDD would be helpful for their differential diagnosis. Since olfactory tests are low-cost and easily accessible, they could be used to screen patients with a suspicion of DLB, in combination with DLB symptoms. Patients that would be considered as suspect after olfactory screening would be referred for deeper investigation, using the other biomarkers previously mentioned, therefore minimizing the use of invasive and expensive tests ${ }^{(23)}$.

The odor test used in this study encompasses three different odorants, know to differentially activate the olfactory and trigeminal chemosensory system ${ }^{(17,18)}$. We found that both olfactory and trigeminal sensitivities (most specifically assessed with respectively PEA and Pyridine) were more impaired in DLB patients than as compared to PPD patients; and in PPD patients as compared to healthy controls. In the present study, we chose Pyridine to stimulate trigeminal system. Since it is known that,

\section{Canonical discriminant functions}

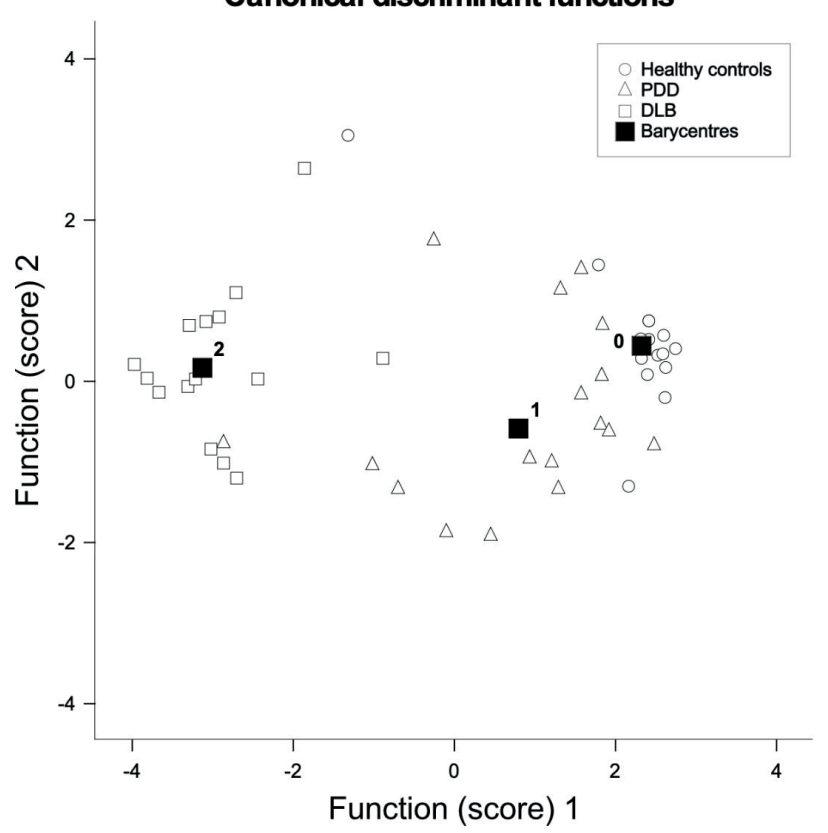

Figure 2. Canonical discriminant functions. Function (score) $1=$ $0.760 *$ PEA + 0.430*n-Butanol + 0.159*Pyridine - 3.966; Function (score) $2=0.029 *$ PEA $+0.203 * n-B u t a n o l-0.175 *$ Pyridine +0.208 . Group 2 (2): Dementia with Lewy bodies (DLB) patients, Group 1 (1): Parkinson disease dementia (PDD) patients, Group 0 (0): Healthy control participants. Acronyms odorants: PEA: Phenyl ethyl alcohol

for a given odorant, odor thresholds are lower than their trigeminal threshold, it can not be excluded that Pyridine threshold reflect at least partly olfactory threshold. However, pyridine have stronger trigeminal (intranasal) components than do more purely olfactory stimuli such as PEA ${ }^{(19,20)}$.

Incidentally, n-Butanol thresholds did not significantly differ between PDD and DLB; the results were close to significance level. This might be explained by n-Butanol's characteristics (mediated both by trigeminal and olfactory sensitivities), generating many intermediate (non-extreme) thresholds results in both of the groups, allowing this above-mentioned $p$-value close to significance level. However, discriminant analyses showed that it was possible to differentiate the three groups of participants (Figure 2), based on two distinct linear functions having as variables the three odor detection thresholds. This finding reinforces the hypothesis that detection thresholds for odorant stimulating both the olfactory and trigeminal systems could be considered as a biomarker of DLB. Finally, previous studies reported a correlation between smell loss and cognitive function ${ }^{(24)}$. However, this was mainly found for odor identification performances (25) (preferably used to assess olfactory disorders in AD). In the present study, we found no impact of MMSE score on odor detection threshold results. This might be explained by the fact that odor detection threshold tasks require less cognitive functions that identification tasks. We must also mention as potential 
bias the non-inclusion of subjects with cognitive impairments in the healthy controls group.

\section{Conclusion}

Assessment of odor detection thresholds, using odorants that activate both olfactory and trigeminal systems, seems to be useful for the differential diagnosis between DLB from PDD. These results suggest that olfactory and trigeminal detection testing could be considered to increase the diagnostic accuracy of DLB, in combination with the classical clinical work-up evaluation. Researches evaluating olfacto-trigeminal interactions in these populations of patients would be of interest to further support our findings.

\section{Acknowledgements}

We would like to thank Dr Gerard Brand with whom this work and the olfactory tests have begun; and Dr Bernard Kamsu
Foguem, Dr Donatien Chedom Fotso and Dr Henry Abanda for their contribution and analyses of the data.

\section{Authorship contribution}

CF: design of project, collection of the data, drafting of the manuscript and revised it; $\mathrm{CF}, \mathrm{ML}, \mathrm{CH}$ : analysis and interpretation of the data; $\mathrm{ML}, \mathrm{CH}$ : critical revision of the manuscript; $\mathrm{CF}, \mathrm{ML}, \mathrm{CH}$ : Approval of the final version of the manuscript.

\section{Conflict of interest}

The authors declare that they have no conflict of interest with regard to this research.

\section{Abbreviations}

SPSS: Statistical Package for the Social Sciences; MRI: magnetic resonance imaging ; PET: positron emission tomography; REM: Rapid eye movement; CSF: cerebrospinal fluid.

\section{References}

1. Foguem C, Kamsu-foguem B Neurodegeneration in tauopathies and synucleinopathies. Rev Neurol (Paris). 2016; 172(11):709-714.

2. McKeith IG, Boeve BF, Dickson DW, et al Diagnosis and management of dementia with Lewy bodies: Fourth consensus report of the DLB Consortium. Neurology. 2017; 89(1):88-100

3. Tröster Al. Neuropsychological characteristics of dementia with Lewy bodies and Parkinson's disease with dementia: differentiation, early detection, and implications for "mild cognitive impairment" and biomarkers. Neuropsychol Rev. 2008; 18(1):103-119.

4. Foguem C, Manckoundia P. Lewy Body Disease: Clinical and Pathological "Overlap Syndrome" Between Synucleinopathies (Parkinson Disease) and Tauopathies (Alzheimer Disease). Curr Neurol Neurosci Rep. 2018; 18(5):24.

5. Nelson PT, Jicha GA, Kryscio RJ, et al. Low sensitivity in clinical diagnoses of Lewy body disease. J Neurol. 2010; 257(3):359366.

6. Henchcliffe C, Dodel R, Beal MF. Biomarkers of Parkinson's disease and Dementia with Lewy bodies. Prog Neurobiol. 2011; 95(4):601-13.

7. Hummel $T$, Whitcroft $K L$, Andrews $P$ et al. Position paper on olfactory dysfunction. Rhinology. 2017;54 (supplement 26): 1-30.

8. Barresi M, Ciurleo R, Giacoppo S, et al. Evaluation of olfactory dysfunction in neurodegenerative diseases. J Neurol Sci. 2012; 323(1-2):16-24.9

9. Hummel T. Assessment of intranasal trigeminal function. Int J Psychophysiol. 2000; 36: 147-55.

10. Mckeith IG, Dickson DW, Lowe J, et al. Consortium on DLB, Diagnosis and management of dementia with Lewy bod- ies: third report of the DLB Consortium. Neurology. 2005;65(12):1863-1872.

11. Dubois B, Burn D, Goetz C, et al. Diagnostic procedures for Parkinson's disease dementia: recommendations from the movement disorder society task force. Mov Disord. 2007; 22(16):2314-2324.

12. Thomas P, Hazif-Thomas C. Dépression, présentation clinique et diagnostic chez la personne âgée. Revue Geriatr. 2003;28:247258.

13. Derouesne C, Poitreneau J, Hugonot L, Kalafat M, Dubois B, Laurent B. Mini-Mental State Examination: a useful method for the evaluation of the cognitive status of patients by the clinician. Consensual French version. Presse Med. 1999; 28 (21):11411148.

14. Bertram L, Tanzi RE. The genetic epidemiology of neurodegenerative disease. J Clin Invest. 2005;115(6):1449-1457.

15. Spering CC, Hobson V, Lucas JA, Menon CV, Hall V, O'Bryant SE. Diagnostic accuracy of the MMSE in detecting probable and possible Alzheimer's disease in ethnically diverse highly educated individuals: an analysis of the NACC database. J Gerontol A Biol Sci Med Sci. 2012; 67(8):890-896.

16. Shulman KI. Clock-drawing: is it the ideal cognitive screening test? Int J Geriatr Psychiatry. 2000;15(6):548-561.

17. Rombaux P, Mouraux A, Bertrand B, Guerit JM, Hummel T. Assessment of olfactory and trigeminal function using chemosensory event-related potentials. Neurophysiol Clin. 2006; 36(2):53-62.

18. Foguem C, Brand G. Comparison of olfactory thresholds between elderly with Parkinson disease and controls. J Aging Gerontol. 2014; 2:5-12.

19. Brand G. Olfactory/trigeminal interactions in nasal chemoreception. Neurosci Biobehav Rev. 2006;30(7):908-917.
20. Foguem C, Lemdani M, Huart C. Parkinson disease in eldery patients: lessons from odour detection thresholds on olfactotrigeminal interaction. Rhinology. 2018; 56 (2):127-132.

21. Ottaviano G, Savietto E, Scarpa B, et al. Influence of number of drugs on olfaction in the elderly. Rhinology. 2018;56(4):351-7.

22. Wang $X$, Zhang C, Xia X, Yang Y, Zhou C. Effect of gender on odor identification at different life stages: a meta-analysis. Rhinology. 2019;57(5):322-30.

23. Donaghy PC, McKeith IG. The clinical characteristics of dementia with Lewy bodies and a consideration of prodromal diagnosis. Alzheimers Res Ther. 2014;6(4):46.

24. Mckeown DA, Doty RL, Perl DP, Frye RE, Simms I, Mester A. Olfactory function in young adolescents with Down's syndrome. J Neurol Neurosurg Psychiatry. 1996; 61(4):412-414.

25. Devanand DP, Tabert MH, Cuasay K, et al. Olfactory identification deficits and $\mathrm{MCl}$ in a multi-ethnic elderly community sample. Neurobiol Aging. 2010; 31(9):1593-1600.

\section{Dr Clovis Foguem}

Auban Moët Hospital

137 rue de l'hôpital, BP: 137

51200 Epernay

France

Tel: 00(33)621120819

Fax: 00(33)326587171

E-mail: cfoguem@yahoo.fr

ORCID iD: 0000-0003-1577-0721 